\title{
Evidencias de manipulación de resultados presupuestarios en el sector público: análisis cross-subnational para el Brasil*
}

\section{Evidence of manipulation of budgetary results in the public sector: Cross-subnational analysis for Brazil}

\author{
Paulo Sérgio Almeida-Santos ${ }^{a}$ \\ Universidade Federal de Mato Grosso, Brasil \\ psalmeidasantos@hotmail.com \\ ORCID: http://orcid.org/0000-0003-1297-7233 \\ Josimar Pires da Silva \\ Universidade Federal da Grande Dourados, Brasil \\ ORCID: http://orcid.org/0000-0002-7769-8748 \\ José Matias-Pereira \\ Universidade de Brasilia, Brasil \\ ORCID: http://orcid.org/0000-0001-6320-026X \\ Augusta Conceição Ferreira dos Santos \\ Universidade de Aveiro, Portugal \\ ORCID: http://orcid.org/0000-0003-1659-3309
}

DOI: https://doi.org/10.11144/Javeriana.cc18-48.emrp

Redalyc: http://www.redalyc.org/articulo.oa?

Fecha de recepción: 09 Marzo 2018

Fecha de aprobación: 18 Octubre 2018 Fecha de publicación: 20 Diciembre 2018

$$
\text { id }=151557795007
$$

\section{Resumen:}

Este artículo indaga sobre los incentivos que podrían tener los gestores públicos de los gobiernos regionales brasileños para manipular los resultados presupuestales como respuesta a los ciclos electorales y a la ley de responsabilidad fiscal. Mediante a un análisis cross-regional se analizan los gobiernos regionales brasileños $(\mathrm{n}=27)$, durante el periodo 1989-2014, para un total de 702 observaciones. Los principales hallazgos indican que los gobiernos se involucran con la manipulación de los resultados presupuestarios, esto es, reportan pequeños presupuestos cercanos a cero. La evidencia muestra, por un lado, que el ciclo electoral no tiene efectos conclusivos sobre esta práctica, y por el otro lado, que los gobiernos tienen incentivos para responder positivamente a las reglas de la ley de responsabilidad fiscal.

Palabras clave: manipulación de resultados, presupuesto público, ciclo electoral, ley de responsabilidad fiscal.

\section{Abstract:}

The aim of the study is to verify the extent to which Brazilian regional governments opportunistically manipulate the budgetary results in the reporting of small balances, and whether the electoral cycles and the fiscal responsibility laws are incentives for this practice. Through a cross-regional analysis, we researched the case of the Brazilian regional governments $(n=27)$, during a longitudinal period from 1989 to 2014, totaling 702 observations. The main discoveries indicate that governments tend to be involved in the manipulation of budgetary results, that is, they disclose small budgetary balances close to zero. Also, note that the electoral cycle has no conclusive effects on this practice; at the same time, the governments have incentives to respond positively to the rules of the fiscal responsibility law. Keywords: earnings management, public budget, electoral cycle, fiscal responsibility law.

\section{Introducción}

Uno de los temas más discutidos en las últimas décadas en el ámbito de la contabilidad se refiere a la manipulación de resultados contables. No obstante, un vacío percibido en este campo de estudio lo constituye el enfoque de la manipulación de resultados en el contexto de las entidades públicas.

Notas de autor

a Autor de correspondencia. Correo electrónico: psalmeidasantos@hotmail.com 
El comportamiento oportunista puede conducir a los gobiernos a manipular las cuentas públicas con el fin de satisfacer, en especial, sus propios intereses. En otras palabras, los gobiernos pueden aumentar sus gastos principalmente para satisfacer sus necesidades de reelección o de mantenimiento del poder partidario, haciendo uso de las áreas más estratégicas de interés de la población, es decir gastos políticamente más visibles por la población (e.g., inversión en infraestructura, gastos en salud, educación, seguridad, etc.) para lograr el éxito.

La teoría de los ciclos políticos, en particular, su vertiente de los ciclos políticos presupuestarios (CPP) (Rogoff \& Sibert, 1988; Rogoff, 1990; Rosenberg, 1992; Brender \& Drazen, 2005; Veiga \& Veiga, 2005), certifica que los gestores públicos tienden a cambiar su comportamiento de búsqueda para aumentar sus posibilidades de ser elegido nuevamente, es decir, que ellos tienden a adoptar una política más expansiva previo a elecciones. En esta perspectiva, si hay un aumento en el gasto público los resultados presupuestarios declarados por los gobiernos mostrarán un alza en los periodos ex ante a las elecciones. Sin embargo, si no se observa este comportamiento puede haber evidencias de manipulación, al menos parcialmente, teniendo cuenta que los resultados negativos bastante acentuados podrían llamar la atención de los órganos de control o de la oposición política. Además, gastos muy acentuados pueden comprometer los límites establecidos por las reglas fiscales. Souza (2008) verificó que en determinadas elecciones los gobiernos regionales brasileños extrapolaron de modo acentuado las metas fiscales exigidas por la ley de responsabilidad fiscal (LRF).

La literatura que discute la contabilidad creativa en el ámbito del sector público, preconiza que bajo las reglas rígidas de leyes de responsabilidad fiscal los gobiernos tienen incentivos para manipular las cuentas públicas, buscando mostrar una realidad ficticia para los resultados presupuestarios a fin de no sufrir, por lo tanto, las penalizaciones de las leyes de delito fiscales ni parte de los órganos de control (Dafflon \& Rossi, 1999; Milesi-Ferretti; 2003; Von-Hagen \& Wolff, 2006; Milesi-Ferretti \& Moriyama, 2006; Bernoth \& Wolff, 2008; Souza, 2008; Maltritz \& Wüste, 2015; Reischmann, 2015).

El objetivo de este artículo consiste en verificar en qué medida los gobiernos regionales brasileños manipulan oportunistamente los resultados presupuestarios en una perspectiva de reportar pequeños saldos, y si los ciclos electorales y la ley de responsabilidad fiscal son incentivos para esta práctica. Así, utilizando pruebas de chi-cuadrado de Pearson, estadística de Cramér's V, y correlación Tetracortica, los resultados indican que existen indicios de manipulación de resultados presupuestarios entre los gobiernos regionales brasileños.

El artículo se organiza en cuatro secciones, aparte de esta introducción. En la primera sección se expone la revisión de la literatura sobre la teoría de los ciclos político-económicos y presupuestarios, y también sobre la gestión de resultados en el sector público, mostrando las hipótesis que buscamos probar. La segunda sección está dedicada a presentar la muestra, describir la metodología utilizada, explicar cómo se construyeron las variables y cómo se analizaron. La tercera sección está destinada a valorar los resultados obtenidos, y en la cuarta y última sección se presentarán las conclusiones del estudio.

\section{Revisión de la literatura}

\section{Manipulación de resultados presupuestarios}

El presupuesto público es una de las principales herramientas administrativas que contribuye para la gestión y transparencia en la aplicación de los recursos públicos. De hecho, es el principal instrumento disciplinario de las finanzas públicas. Por lo tanto, en la planeación y en la ejecución se deben seguir algunos principios normativos, exigidos por reglamentos contables y fiscales, entre ellos, citase la búsqueda por el equilibrio de las cuentas públicas (Santos et ál., 2012). 
En líneas generales, el resultado presupuestario se determina como la diferencia entre los ingresos y los gastos del periodo. Los resultados son superavitarios si los ingresos superan los gastos presupuestarios; y en cambio, los resultados son deficitarios, si los gastos superan los ingresos presupuestarios; y nulo o equilibrado, si los ingresos y gastos presupuestarios tienen igual importe. El estudio del resultado presupuestario es oportuno, pues ello "ocupa un lugar destacado en la contabilidad pública al aportar evidencias sobre la viabilidad financiera de las administraciones públicas y sobre la actuación de los responsables de las mismas en este ámbito" (González \& Velázquez, 2015, p. 108). Además, el presupuesto es, con efecto, una "arena” ${ }^{1}$ por la disputa de recursos públicos (Azevedo, 2013), y, por lo tanto, mediante la política fiscal, los gobiernos tienen motivaciones para manipular los agregados presupuestarios, especialmente cuando estos buscan mantenerse en el poder (Ferreira et ál., 2013; Reischmann, 2015).

Es escasa la literatura sobre la manipulación de resultados (earnings management) en el sector público. Para los investigadores resulta difícil trasponer los modelos seminales de la literatura (Healy, 1985; Schipper, 1989; Jones, 1991; Dechow \& Sloan, 1995) para el contexto de las entidades públicas, especialmente porque estos modelos se basan en la contabilidad sobre una base de acumulación (accruals), algo que todavía es nuevo para muchas entidades públicas en todo el mundo (Stalebrink, 2002 y 2007; Ferreira et ál., 2013; Pellicer et ál., 2013).

Stalebrink (2002 y 2007) al investigar el caso de los municipios suecos, verificó que estos elevan sus costos con depreciación y amortización cuando tienen una tendencia a presentar resultados económico-patrimonial positivos, y disminuyen esos mismos costos cuando buscan presentar resultados económico-patrimonial negativos. Ferreira et ál. (2013) estudian las autarquías portuguesas y demuestran que los gestores intentan actuar de modo oportunista, i.e., buscan maximizar su bienestar, evitando divulgar resultados económicopatrimonial negativos, así, divulgan resultados económico-patrimonial cerca de cero, buscando señalar un óptimo desempeño ante a los electores en la tentativa de aumentar sus votos. Pellicer et ál. (2013) también demuestran que los municipios ingleses tienden a mantener sus resultados económico-patrimonial cerca de cero. Las cuentas de depreciación y amortización son las más utilizadas para esta finalidad.

Sin embargo, estas investigaciones verifican el oportunismo de los gestores públicos sobre el resultado económico-patrimonial, lo que motiva investigaciones al respecto de la manipulación del resultado presupuestario, que desde la perspectiva de la política fiscal a veces ha sido investigado bajo la temática de la contabilidad creativa.

Por ejemplo, Santos et ál. (2012) buscan identificar municipios brasileños pertenecientes a la Federación de Santa Catarina, manipulando sus resultados presupuestarios mediante la fuente de otros ingresos corrientes (e.g., multas, intereses) y otros gastos corrientes (e.g., material de consumo, gastos de mantenimiento). Las evidencias indican que las dos fuentes fueron manipuladas de manera oportunista. Más precisamente, las dos fuentes están correlación, y tienden a disminuir cuando el resultado presupuestario tiene una variación negativa. Si los ingresos tienden a disminuir el resultado presupuestario, los gastos también siguen el mismo camino. Además, se contraen los otros ingresos y los gastos corrientes, así, el resultado presupuestario es positivo en años electorales.

En el caso de Brasil, estudios anteriores, muestran que de algún modo los gestores tienden a manipular los agregados presupuestarios para atender, sobre todo, límites de gastos constitucionales y también de ejecuciones fiscales: gastos de salud (Dalmonech et ál., 2008; Queiroz \& Rodrigues, 2014); gastos de educación (Dalmonech et ál., 2008); los gastos de personal (Queiroz et ál., 2013); y resultados fiscales (Gobetti, 2006; Flach, 2012; Scaff \& Athias, 2016; Gobetti \& Orair, 2017; Paganotto et ál., 2017).

Por lo demás, a partir de la literatura se puede deducir que los gobiernos pueden tener dos incentivos para manipular sus resultados presupuestarios: el primero es la motivación electoral, para alcanzar el mayor número de votos posibles, por ende los gobiernos tendrían motivaciones para manipular los agregados presupuestarios a fin de señalar que tiene compromiso con la sociedad, especialmente aumentando el nivel de gasto políticamente más visible por la población. La segunda motivación está relacionada con determinados 
objetivos contables y/o fiscales de los gobiernos para demostrar competencia administrativa y técnica, tanto a la población como a los órganos de control y/o demás interesados.

\section{Motivaciones electorales}

Las motivaciones electorales han sido estudiadas por la teoría de la elección pública, especialmente por de la corriente teórica de los ciclos político-económicos y político-presupuestarios. La teoría de la elección pública tiene como premisa general que los gobiernos no son altruistas, por lo tanto, buscan sus propios intereses, y tienden a comportarse como cualquier otro agente del mercado, siempre maximizando su utilidad, así, los gobiernos también son homos economicus en el mercado político (Pereira, 1997).

El estudio seminal de Nordhaus (1975) dio partida a las discusiones empíricas sobre el oportunismo político (ciclos político-económicos) mostrando que los gobiernos tienden a manipular variables macroeconómicas cuando se acercan las elecciones, más precisamente existe la presencia de un trade-off entre la tasa de empleo y de inflación. Este modelo de Nordhaus (1975) es denominado de modelo oportunista. Además, tenemos el modelo partidario (Hibbs Jr., 1977); el modelo oportunista con expectativas racionales (Persson \& Tabellini, 1990); y el modelo partidario con expectativas racionales (Alesina, 1987).

No obstante, la falta de evidencias empíricas más consistentes en lo que se refiere la literatura de los ciclos político-económicos, tiene inducido el cambio en el foco de los ciclos políticos, principalmente, con relación a la expansión fiscal de los gastos públicos, o llamado, ciclos político-presupuestarios (Brender \& Drazen, 2005). Para los gobiernos es más fácil manipular las variables presupuestarias que las variables económicas: tasa de empleo, inflación, etc. (Veiga \& Veiga, 2005), especialmente gobiernos de esferas más pequeñas, e.g., gobiernos regionales, municipales, etc.

La principal perspectiva de los ciclos políticos-presupuestarios está en el análisis del oportunismo gubernamental sobre los gastos públicos, en que al buscar la reelección ellos tienden a adoptar una política más expansionista cuando se acercan las elecciones, y a fin de compensar estos gastos oportunistas, utilizan una política más construccionista después del proceso electoral (Rogoff \& Sibert, 1988; Rogoff, 1990; Rosenberg, 1992). Ver la figura 1.

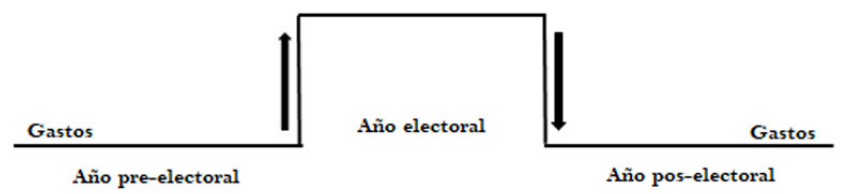

FIGURA 1. Dinámica de los gastos en el contexto de los ciclos político-presupuestarios

Fuente: adaptado de Pennings et ál. (2006, p. 278).

En este contexto, el ambiente electoral se vuelve más oportuno para la investigación de la manipulación de resultados en el sector público. Ferreira et ál. (2013) encontraron relación entre periodos pre-electorales y la manipulación de resultados en la perspectiva de breakeven relativo al resultado económico-patrimonial. Reischmann (2015) afirma que las motivaciones electorales pueden llevar a los gestores públicos a involucrarse fuertemente con la manipulación de resultados presupuestarios. Para el caso de los países de OCDE, el autor encuentra evidencias que sugieren que los gobiernos se involucran estratégicamente con la manipulación antes de las elecciones, buscando especialmente "disfrazar" el resultado presupuestario (Reischmann, 2015). Ferreira et ál. (2013) apoyan la hipótesis de que la manipulación de resultados en periodos electorales se lleva a cabo por entidades públicas para destacar su rendimiento y competencia, donde los políticos se aprovechan de este oportunismo con miras a la reelección, i.e., buscando maximizar sus votos; por lo tanto, los gestores tendrían motivaciones para generar una realidad ficticia en términos de resultados presupuestarios antes de las elecciones (Milesi-Ferreti, 2003). En este sentido se platea la siguiente hipótesis:

\section{H1: El ciclo electoral está asociado positivamente con la manipulación de los resultados presupuestarios.}


Si por un lado los gobiernos tienden a elevar los gastos políticamente más visibles por la población a fin de obtener votos; por otro, tienen incentivos para no llamar la atención de los órganos de fiscalización/control en razón de potenciales déficits muy acentuados debido al aumento oportunista de los gastos en periodos cercanos a las elecciones, y así, buscarían optimizar el resultado fiscal dentro de los límites establecidos por los reglamentos financieros y/o fiscales (Reischmann, 2015). La investigación de Souza (2008) sobre los estados brasileños encontró evidencias de contabilidad creativa en las elecciones de 2002 y 2006. El autor relata que los informes contables-presupuestarios de los entes al final de estos años era de extremo celo y rigor contable, los ingresos iguales a los gastos, y los grandes totales siempre en equilibrio.

\section{Motivaciones fiscales}

Otro incentivo que llevan a las entidades públicas a manipular sus cuentas se debe, especialmente, a las reglas fiscales, es decir, al esfuerzo continuo para atender ciertas restricciones presupuestarias establecidas por leyes, decretos, reglamentos, etc. Las reglas fiscales buscan establecer el equilibrio de las cuentas públicas, especialmente en lo que se refiere al control de déficits presupuestarios, que llevan a las entidades públicas a contraer deudas; por lo tanto, estas normas buscan entre otros objetivos principales, restringir el comportamiento de los gobiernos (Von-Hagen \& Wolff, 2006).

Los mecanismos de control establecidos por las reglas fiscales tienen entre otros objetivos, limitar el poder discrecional de los gestores sobre el gasto público, buscando disminuir, sobre todo, potenciales déficits presupuestarios que pueden afectar el endeudamiento público, preservándose así la situación fiscal de los entes públicos (Dafflon \& Rossi, 1999; Milesi-Ferretti, 2003; Von-Hagen \& Wolff, 2006; Milesi-Ferretti \& Moriyama, 2006; Bernoth \& Wolff, 2008; Maltritz \& Wüste, 2015; Reischmann, 2015).

En Brasil, la ley de responsabilidad fiscal (LRF) aprobada bajo la Ley Complementaria 101/2000, "estableció un marco importante en la gestión de los recursos públicos en término de métodos para mejorar la eficiencia de la gestión pública y la sostenibilidad de la capacidad de inversión de ese sector" (Santos, 2007, p. 22). Su objetivo primordial es establecer las normas de finanzas públicas dirigidas a la responsabilidad en la gestión fiscal y de otras providencias, estableciendo que los siguientes objetivos sean alcanzados por todas las entidades públicas nacionales: a) la acción planificada y transparente; b) la prevención de riesgos, así como la corrección de desvíos capaces de afectar el equilibrio de las cuentas públicas; c) el cumplimiento de metas de resultados entre ingresos y gastos; d) los límites y condiciones en lo que se refiere: la renuncia de ingresos, generación de gastos de personal, seguridad social, deudas consolidadas y mobiliarias, operaciones de crédito, concesión de garantía e inscripción en restos a pagar.

A pesar de la existencia de las normas fiscales, especialmente las leyes de responsabilidad fiscal que, en particular, obligan a los organismos públicos a mantener el equilibrio de sus cuentas (Milesi-Ferretti, 2003; Milesi-Ferretti \& Moriyama, 2006; Maltritz \& Wüste, 2015), los gobiernos podrían buscar manipular el saldo presupuestario. Según Milesi-Ferreti (2003) las reglas fiscales son incentivos para la llamada contabilidad creativa en el sector público, es decir, los gobiernos pueden cambiar los gastos fuera del presupuesto fiscal, para eludir esas reglas, no sufriendo, pues, sus penalidades y también de las entidades de fiscalización/control.

Aunque las normas fiscales aumentan el nivel de transparencia de las cuentas públicas, así como la inexistencia de la mejor gestión de los recursos públicos, en medidas umbrales, sobre todo para el control del endeudamiento y los gastos de personal, etc., y además de ser uno de los instrumentos importantes para el avance de las prácticas de la accountability en el país, puede considerarse un incentivo para que los gestores públicos locales manipulen la información presupuestaria para cumplir los límites establecidos por la LRF. Así, en el intento de ajustar las finanzas de la entidad, los gestores públicos pueden hacer el uso de manipulaciones contables a fin de optimizar los resultados fiscales. Ante las perspectivas que se presentan, se establece la siguiente hipótesis: 
H2: La ley de responsabilidad fiscal (LRF) está asociada positivamente con la manipulación de los resultados presupuestarios.

A pesar de la severidad de las reglas fiscales, los gobiernos pueden actuar de manera oportunista en la gestión de los gastos públicos bordeando los límites legales preestablecidos, por ejemplo, para determinados gastos, déficit, endeudamiento, etc., luego las reglas fiscales son un incentivo que pueden llevar a las entidades públicas a manipular los resultados presupuestarios. Por lo tanto, los gobiernos pueden manipular los agregados presupuestarios para "disfrazar" el resultado fiscal (Reischmann, 2015). En diversas situaciones los gestores pueden utilizar vacíos de las leyes de responsabilidad fiscal a fin de dar otro destino a las cuentas públicas (Souza, 2008), especialmente en su último año de mandato (Milesi-Ferreti, 2003).

\section{Método}

\section{La muestra y los datos}

La muestra investigada comprende los Estados brasileños más el Distrito Federal $(\mathrm{n}=27)$. Los datos son referentes a los años 1989 a 2014 ( $\mathrm{T}=28$ ); y un total de ocho periodos electorales: 1990, 1994, 1998, 2002, 2006, 2010 y 2014. La fuente de los datos son la Secretaría del Tesoro Nacional y el Tribunal Superior Electoral. En lo que se refiere exclusivamente a los datos financieros, la fuente son las bases de datos SISTN y FINBRA. Los dados financieros son relativos a los precios constantes de 2014, corregido por el Índice General de Precios (IGP-DI) de la Fundação Getúlio Vargas (FGV).

\section{Medición de la manipulación de resultados presupuestarios}

Considerando que las entidades del sector público trabajan con instrumentos de planificación, los cuales orientan la ejecución de los ingresos y gastos públicos a fin de buscar y mantener el equilibrio entre estos; es común que presenten resultados equilibrados, especialmente cercanos a cero. Además, la fuerte presión de la LRF para alcanzar el equilibrio fiscal es fundamental para que los gobiernos mantengan la ejecución de sus presupuestos alineados a lo planeado, aunque los déficits presupuestarios son recurrentes, sobre todo, en años electorales en que comprobadamente los gastos públicos sufren grandes elevaciones, según testifica la teoría de los ciclos político-presupuestarios.

Inicialmente se calculó el nivel de variación de los resultados presupuestarios utilizando la siguiente formulación:

$$
\Delta R P_{i, t}=\frac{I_{i t}-G_{i t}}{I_{i t-1}}
$$

Donde $\triangle R P_{i t}$ es la variación del resultado presupuestario; $i_{i t}$ es los ingresos presupuestarios; $G_{i t}$ son los gastos presupuestarios. Luego se hizo la estandarización de $\triangle R P_{i t}$, por medio de $Z$-score:

$$
Z_{i t}=\frac{x_{i}\left(\Delta R P_{i t}\right)-\mu\left(\Delta R P_{i t}\right)}{\sigma\left(\Delta R P_{i t}\right)}
$$


Así, se define la variable MRP (manipulación de resultados presupuestarios) con los valores $(Z$-score) dentro del rango entre -0.10000 y 0.10000 . Se partió de la premisa de que si las entidades públicas tienen motivaciones para manipular los resultados presupuestarios, ellos buscarían mantener sus resultados a cerca de cero, es decir:

$$
\mathrm{MRP}=\left\{\begin{array}{l}
\left.\left.1, \operatorname{si} Z\left(\Delta R P_{i t}\right)=\right]-0.10000 ; 0.10000\right] \\
\left.\left.0, \operatorname{si} Z\left(\Delta R_{i, t}\right) \neq\right]-0.10000 ; 0.10000\right]
\end{array}\right.
$$

Este procedimiento es similar al utilizado por Burgstahler y Dichev (1997) para el caso de empresas privadas, y por Ferreira et ál. (2013), Callahan y Waymire (2015) para el caso de entidades públicas. Por consiguiente, se verifica la asociación entre la MRP y el ciclo electoral y también la LRF, utilizándose tablas cruzadas por medio de la prueba de chi-cuadrado de Pearson, la prueba de Cramér's $V$, y la correlación Tetracortica.

\section{Resultados y discusión}

En la figura 2 se ilustra el histograma de la distribución cross-section de frecuencia de los resultados presupuestarios normalizados (pequeños resultados). En líneas generales, siguiendo la metodología propuesta en la sección anterior, se observa que la manipulación de los resultados corrientes es la más frecuente entre las entidades públicas analizadas $(\mathrm{n}=104)$; la evidencia de manipulación inherente a los resultados de capital se registra sólo para 54 casos; y la manipulación del resultado general tiene evidencia para 81 casos, de un total de 702 observaciones. En lo que se refiere al relato de pequeñas variaciones de los resultados presupuestarios ilustrados en la figura 2, se percibe un comportamiento similar al encontrado por Callahan y Waymir (2015) para el caso de las grandes ciudades norteamericanas. Para estas autoras, cuando los gestores públicos muestran pequeños saldos presupuestarios están respondiendo a determinados incentivos para la creación de holguras presupuestarias o determinadas metas de resultados.

En la figura 2 se puede observar que los pequeños resultados del presupuesto corriente tienen una fuerte correlación positiva con los pequeños resultados del presupuesto general $\left(r_{x y}=0,747 ; p<1 \%\right)$; mientras que los pequeños resultados del presupuesto de capital se correlacionan de manera moderada y positiva con los pequeños resultados del presupuesto general $\left(r_{x y}=0,495 ; p<1 \%\right)$. En esta dirección, se plantea que, en general, las variaciones del resultado presupuestario general de los entes regionales brasileños son respondidas por las variaciones de los recursos corrientes.

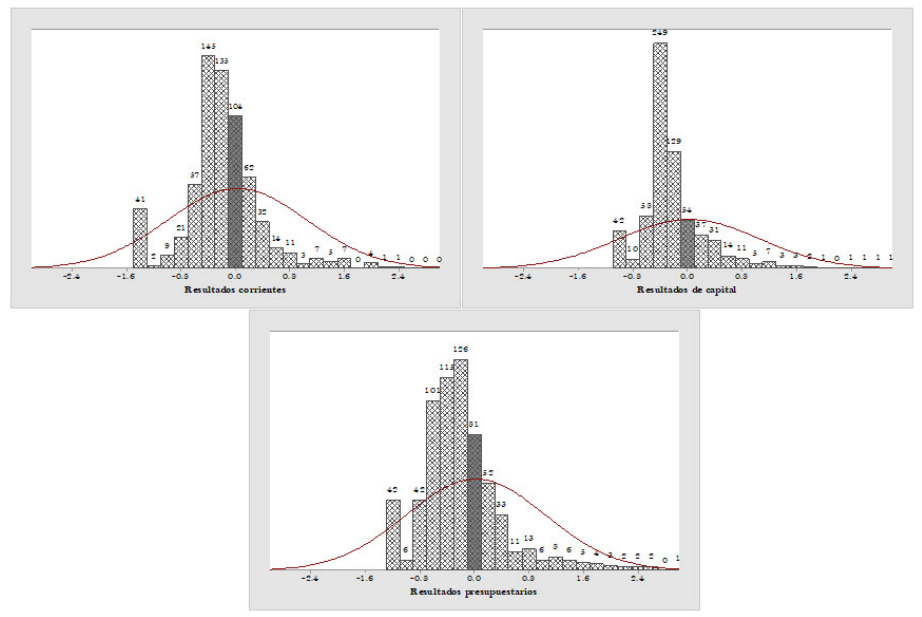

FIGURA 2. Histograma de la distribución cross-section de frecuencia de los resultados presupuestarios normalizados Fuente: elaboración propia. 
En la tabla 1 se presentan las estadísticas descriptivas de las frecuencias relativas a las evidencias de manipulación de resultados presupuestarios a lo largo de la serie. Un hecho que se destaca es el efecto de la ley de responsabilidad fiscal, pues a partir del año de su aprobación (2000) se percibe un aumento en las evidencias de manipulación de resultados presupuestarios entre las entidades públicas investigadas, cuya mayor frecuencia, de modo especial, puede ser vista con relación a los resultados corrientes. Esto se justifica por el fuerte impacto de la LRF sobre las cuentas públicas locales (Santos \& Alves, 2011), y especialmente por reducir los déficits presupuestarios (Souza, 2008).

TABLA 1

Estadísticas descriptivas de las frecuencias relativas a la evidencia de manipulación de los resultados presupuestarios en la serie

\begin{tabular}{|c|c|c|c|c|}
\hline & $\begin{array}{c}\text { Resultados } \\
\text { corrientes }\end{array}$ & Resultados de capital & Resultados general & $F i$ \\
\hline 1989 & 4 & 2 & 5 & 11 \\
\hline $1990^{\neq}$ & 1 & 1 & 0 & 2 \\
\hline 1991 & 2 & 3 & 3 & 8 \\
\hline 1992 & 6 & 1 & 5 & 12 \\
\hline 1993 & 3 & 5 & 5 & 13 \\
\hline $1994^{*}$ & 1 & 2 & 1 & 4 \\
\hline 1995 & 3 & 1 & 1 & 5 \\
\hline 1996 & 0 & 3 & 1 & 4 \\
\hline 1997 & 3 & 4 & 1 & 8 \\
\hline $1998^{\neq}$ & 2 & 1 & 3 & 6 \\
\hline 1999 & 2 & 3 & 0 & 5 \\
\hline $2000^{\dagger}$ & 0 & 0 & 0 & 0 \\
\hline 2001 & 6 & 0 & 3 & 9 \\
\hline $2002^{ \pm}$ & 9 & 4 & 7 & 20 \\
\hline 2003 & 4 & 0 & 3 & 7 \\
\hline 2004 & 6 & 3 & 8 & 17 \\
\hline 2005 & 7 & 1 & 6 & 14 \\
\hline $2006^{ \pm}$ & 8 & 2 & 4 & 14 \\
\hline 2007 & 7 & 0 & 4 & 11 \\
\hline 2008 & 3 & 3 & 7 & 13 \\
\hline 2009 & 8 & 1 & 5 & 14 \\
\hline $2010^{ \pm}$ & 11 & 5 & 4 & 20 \\
\hline 2011 & 3 & 1 & 2 & 6 \\
\hline 2012 & 3 & 3 & 3 & 9 \\
\hline 2013 & 0 & 2 & 0 & 2 \\
\hline $2014^{\neq}$ & 2 & 3 & 0 & 5 \\
\hline & & & & 23 \\
\hline Global & 104 & 54 & 81 & 9 \\
\hline
\end{tabular}

En la tabla 2 se presentan las estadísticas relativas a la asociación entre el periodo preelectoral y la manipulación de resultados presupuestarios. El periodo preelectoral está asociado positivamente con la manipulación de los resultados corriente y general; y negativamente asociado con los resultados de capital. Sin embargo, se resalta que las pruebas estadísticas no son significativas hasta el nivel máximo establecido, i.e., 10\%. Estos resultados confirman parcialmente las conclusiones de Reischmann (2015) y Souza (2008). Así, si por un lado en periodos preelectorales los gobiernos regionales brasileños tienen incentivos para aumentar 
la manipulación del resultado del presupuesto corriente y general, por otro, tiene incentivos para disminuir la del presupuesto de capital.

TABLA 2

Ciclo electoral versus manipulación de resultados presupuestarios: periodos preelectorales

\begin{tabular}{|c|c|c|c|c|c|}
\hline Resultado corriente & & & & & \\
\hline \multirow{5}{*}{ ¿Evidencia de manipulación? } & \multicolumn{4}{|c|}{ ¿Periodo preelectoral? } & Pruebas \\
\hline & & No & Sí & Total & \multirow{4}{*}{$\begin{array}{c}\text { Pearson chi2 }=0.5163 \\
\text { Cramér's } V=0.0271 \\
\text { Tetrachoric rho }=0.0550\end{array}$} \\
\hline & No & 440 & 158 & 598 & \\
\hline & Sí & 73 & 31 & 104 & \\
\hline & Total & 513 & 189 & 702 & \\
\hline \multicolumn{6}{|l|}{ Panel B: Resultado de capital } \\
\hline \multirow{5}{*}{ ¿Evidencia de manipulación? } & \multicolumn{4}{|c|}{ ¿Periodo preelectoral? } & Pruebas \\
\hline & & No & Sí & Total & \multirow{4}{*}{$\begin{array}{c}\text { Pearson chi } 2=0.0654 \\
\text { Cramér's } V=-0.0097 \\
\text { Tetrachoric rho }=-0.0241\end{array}$} \\
\hline & No & 473 & 175 & 648 & \\
\hline & Sí & 40 & 14 & 54 & \\
\hline & Total & 513 & 189 & 702 & \\
\hline \multicolumn{6}{|l|}{ Panel B: Resultado general } \\
\hline \multirow{5}{*}{ ¿Evidencia de manipulación? } & \multicolumn{4}{|c|}{ ¿Periodo preelectoral? } & Pruebas \\
\hline & & No & Sí & Total & \multirow{4}{*}{$\begin{array}{c}\text { Pearson chi2 }=0.7229 \\
\text { Cramér's } V=0.0321 \\
\text { Tetrachoric rho }=0.0690\end{array}$} \\
\hline & No & 457 & 164 & 621 & \\
\hline & Sí & 56 & 25 & 81 & \\
\hline & Total & 513 & 189 & 702 & \\
\hline
\end{tabular}

Fuente: elaboración propia.

En la tabla 3 se presentan las estadísticas relativas a la asociación entre el periodo electoral y la manipulación de resultados presupuestarios. El periodo electoral está asociado positivamente con la manipulación de los resultados corriente y de capital; y negativamente asociado con el resultado general. Sin embargo, se resalta que las pruebas estadísticas son significativas sólo para el caso del resultado de capital. Estos resultados también confirman parcialmente las conclusiones de Reischmann (2015) y de Souza (2008). Así, se puede deducir que, debido al uso más agresivo de la política presupuestaria en favor del aumento de gastos políticamente más visibles por la población para obtener votos, los gestores tienden a involucrarse de manera más significativa con la manipulación de los resultados del presupuesto de capital. 
TABLA 3

Ciclo electoral versus manipulación de resultados presupuestarios: periodos electorales

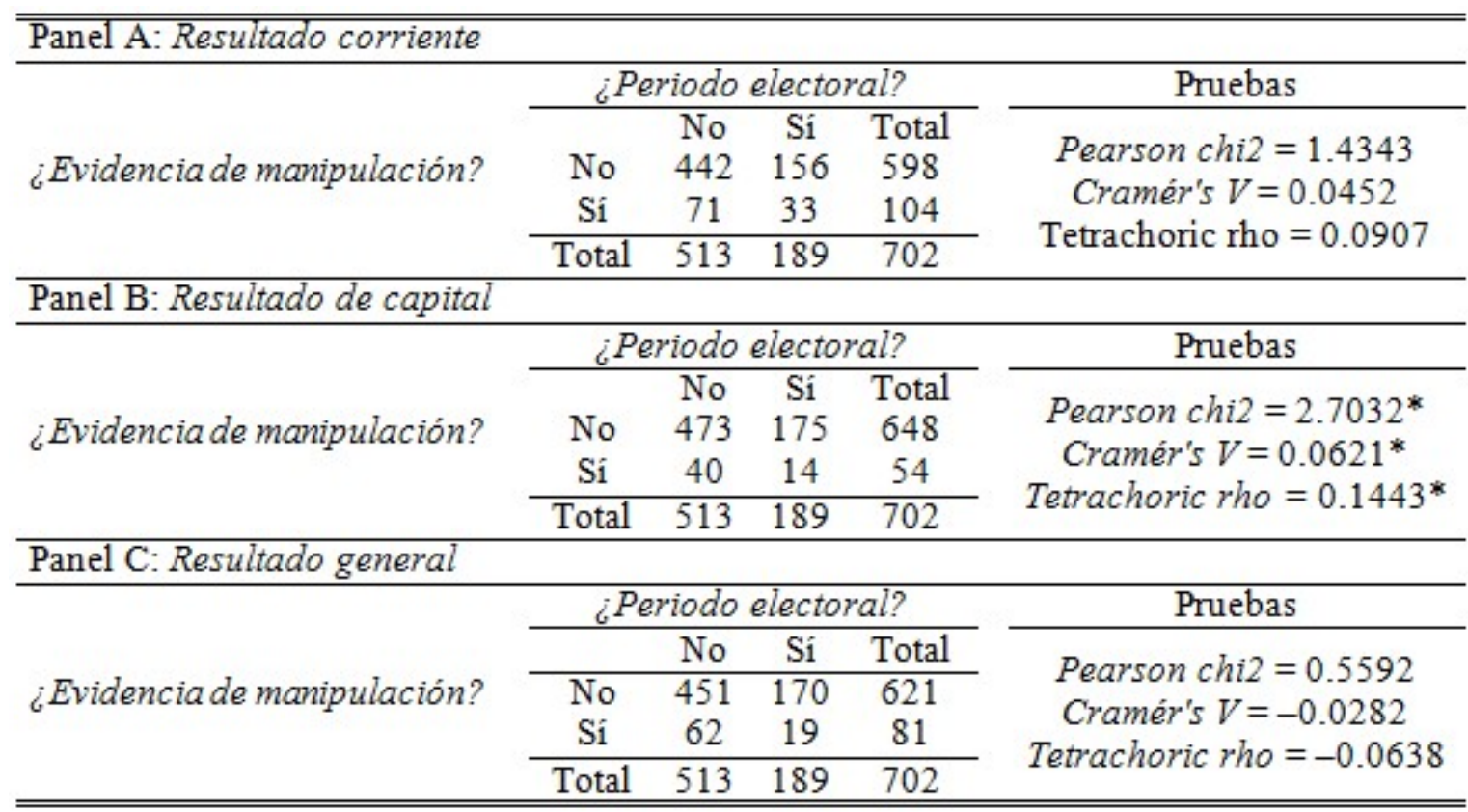

Fuente: elaboración propia.

${ }^{*}$ Significativo al nivel de $10 \%$.

En la tabla 4 se presentan las estadísticas relativas a la asociación entre la ley de responsabilidad fiscal (LRF) y la manipulación de resultados presupuestarios. Con excepción del resultado de capital, la LRF presenta una asociación positiva y estadísticamente significativa con la manipulación de resultados presupuestarios. Así, ante el efecto de las reglas fiscales se puede inferir que los gobiernos regionales brasileños tienden a manipular los resultados presupuestarios; en este caso, buscando reportar pequeños saldos presupuestarios a fin de señalar mayor grado de responsabilidad, especialmente, a los órganos control, evitando, así, posibles sanciones por crímenes de responsabilidad fiscal. Estos resultados son consistentes con los hallazgos de estudios anteriores. 
TABLA 4

LRF versus manipulación de resultados presupuestarios

\begin{tabular}{|c|c|c|c|c|c|}
\hline \multicolumn{6}{|l|}{ Panel A: Resultado corriente } \\
\hline \multirow{5}{*}{ ¿Evidencia de manipulación? } & \multicolumn{4}{|c|}{ ¿Efecto de la LRF? } & Pruebas \\
\hline & & No & Sí & Total & \multirow{4}{*}{$\begin{array}{c}\text { Pearson chi2 }=18.1677^{* * *} \\
\text { Cramér's } V=0.1609 * * * \\
\text { Tetrachoric rho }=0.3163^{* * *}\end{array}$} \\
\hline & No & 296 & 302 & 598 & \\
\hline & Sí & 28 & 76 & 104 & \\
\hline & Total & 324 & 378 & 702 & \\
\hline \multicolumn{6}{|l|}{ Panel B: Resultado de capital } \\
\hline \multirow{5}{*}{ ¿Evidencia de manipulación? } & \multicolumn{4}{|c|}{ ¿Efecto de la LRF? } & Pruebas \\
\hline & & No & Sí & Total & \multirow{4}{*}{$\begin{array}{c}\text { Pearson chi2 }=0.2071 \\
\text { Cramér's } V=-0.0172 \\
\text { Tetrachoric rho }=-0.0395\end{array}$} \\
\hline & No & 473 & 175 & 648 & \\
\hline & Sí & 40 & 14 & 54 & \\
\hline & Total & 513 & 189 & 702 & \\
\hline \multicolumn{6}{|l|}{ Panel C: Resultado general } \\
\hline \multirow{5}{*}{ ¿Evidencia de manipulación? } & \multicolumn{4}{|c|}{ ¿Efecto de la LRF? } & Pruebas \\
\hline & & No & Sí & Total & \multirow{4}{*}{$\begin{array}{c}\text { Pearson chi } 2=8.6132 * * * \\
\text { Cramér's } V=0.1108^{* * *} \\
\text { Tetrachoric rho }=0.2325^{* * *}\end{array}$} \\
\hline & No & 299 & 322 & 621 & \\
\hline & Sí & 25 & 56 & 81 & \\
\hline & Total & 324 & 378 & 702 & \\
\hline
\end{tabular}

Fuente: elaboración propia.

${ }^{* * *}$ Significativo al nivel de $1 \%$.

Los resultados encontrados respaldan la segunda hipótesis, y parcialmente la primera. En el caso de la primera hipótesis: "el ciclo electoral está asociado positivamente con la manipulación de los resultados presupuestarios", se puede concluir que los gobiernos tienen incentivos para relatar pequeños saldos presupuestarios ante el ciclo electoral, siendo más común reportar pequeños saldos para el caso del presupuesto corriente, tanto en periodos preelectorales como electorales. Posiblemente esto se debe al hecho de que el resultado del presupuesto corriente está muy cerca del concepto de resultado primario (i.e., diferencia entre ingresos y gastos del gobierno, excluyendo de la cuenta los ingresos y gastos con intereses). $\mathrm{Y}$ en este sentido, metas establecidas para el resultado primario en la planificación presupuestaria del gobierno, debe ser puntualmente cumplida, siendo que el no cumplimiento de esta meta, es pasivo de penalización por la LRF y demás leyes de crímenes de responsabilidad fiscal. Y así, cuando se analiza la segunda hipótesis: "la LRF está asociada positivamente con la manipulación de los resultados presupuestarios”, se encuentra una fuerte asociación del efecto de la ley de responsabilidad fiscal sobre la manipulación de resultados del presupuesto corriente. Por lo tanto, partiendo del supuesto de que los gestores públicos responden a ese incentivo de regulación fiscal, ellos tendrían motivaciones para manipular preferencialmente los saldos corrientes a capital, y, por consiguiente, el saldo del presupuesto general, posiblemente por considerar que, los saldos corriente y general están más fuertemente correlacionados.

\section{Conclusiones}

Se identificó que los gobiernos regionales brasileños tienden a involucrarse con manipulación de resultados presupuestarios, y que los resultados corrientes son los más manipulados. Los hallazgos del estudio indican también que existe una asociación entre el ciclo electoral y los resultados presupuestarios. Sin embargo, aunque estos resultados no sean concluyentes para todos los resultados presupuestarios analizados; en relación con el saldo del presupuesto de capital, se puede concluir que con el fin de maximizar sus votos, los gobiernos utilizan su poder discrecional para manipular los gastos públicos políticamente más visibles por la población, 
elevándolos, especialmente en los periodos electorales, medida, que, por lo cual, puede impactar el resultado presupuestario. Además, los gobiernos actúan así también por mostrar competencia ante la población, dando la idea de preocupación por el bienestar de la sociedad, con el propósito de mantenerse en el poder, esto es, buscando la reelección.

El interés de los gobiernos por manipular los agregados presupuestarios puede ser doble: de un lado para mostrarle a la población que es un gobierno preocupado por su bienestar; y por otro lado, demostrarle a los órganos de control y a la oposición que el suyo es un gobierno responsable con la gestión de las finanzas públicas. Por lo tanto, se observa también que la ley de responsabilidad fiscal es un fuerte incentivo para que los gobiernos manipulen los resultados presupuestarios para mostrar unas cuentas públicas sanas, es decir, ante rígidas reglas establecidas por la ley de responsabilidad fiscal, los gestores públicos se muestran más cautelosos en señalar, por ejemplo, grandes resultados deficitarios, en efecto, ellos sólo estarían respondiendo particularmente a ese incentivo de control fiscal de las cuentas públicas.

En líneas generales, el estudio además de contribuir a la literatura acerca de la manipulación de resultados en el sector público, también corrobora algunos de los resultados encontrados anteriormente acerca de la asociación entre la manipulación de resultados y los incentivos del ciclo electoral y de las reglas fiscales (Ferreira et ál., 2013; Souza, 2008; Reischmann, 2015). Por lo tanto, se espera que el estudio pueda contribuir a las investigaciones académicas en lo que compete a esta temática. Así como, auxiliar técnicamente a los órganos de control en lo que se refiere a una mejor fiscalización de las cuentas públicas, e igualmente, a la sociedad mediante la instrumentalización del control social a fin de reducir la asimetría de información que de algún modo pueda llevar los gobiernos regionales brasileños a involucrarse con la manipulación de los resultados presupuestarios.

Por último, futuras investigaciones pueden profundizar en otros incentivos además del ciclo electoral y la ley de responsabilidad fiscal, que puedan estar asociados con las prácticas de manipulación de los resultados presupuestarios del sector público.

\section{Referencias}

Alesina, A. (1987). Macroeconomic policy in a two-party system as a repeated game. Quarterly Journal of Economics, 102, 651-678. DOI: https://doi.org/10.2307/1884222

Azevedo, R. (2013). Imprecisão na estimação orçamentária dos municípios brasileiros. Master's thesis, Universidade de São Paulo, Ribeirão Preto, Brazil.

Becker, J. (2015). Estatística básica: transformando dados em informação. Bookman.

Bernoth, K., \& Wolff, G. (2008). Fool the markets? Creative accounting, fiscal transparency and sovereign risk premia. Scottish Journal of Political Economy, 55(4), 465-487. DOI: https://doi.org/10.1111/j.1467-9485.2008.00462 .x

Brender, A. \& Drazen. A. (2005). Political budget cycles in new versus established democracies. Journal of Monetary Economics, 52(7), 1271-1295. DOI: 10.3386/w10539

Burgstahler, D., \& Dichev, I. (1997). Earnings management to avoid earnings decreases and losses. Journal of Accounting and Economics, 24, 99-126. DOI: https://doi.org/10.1016/S0165-4101(97)00017-7

Callahan, C., \& Waymire, T. (2015). The GASB No. 34 impact of budget-to- actual variances on bond ratings: Evidence from U.S. Cities. Journal of Governmental \& Nonprofit Accounting, 4, 32-52. DOI: https://doi.org/10.2308/o gna-51001

Cefaï, D. (2002). ¿Qué es una arena pública? Algunas pautas para un acercamiento pragmático. En Cefaï, D. y Joseph, I. (coords.), La herencia del pragmatismo. Conflictos de urbanidad y pruebas de civismo. París: Edición de l'Aube.

Dafflon, B., \& Rossi, S. (1999). Public accounting fudges towards EMU: A first empirical survey and some public choice considerations. Public Choice, 101(1), 59-84. DOI: https://doi.org/10.1023/A:1018311911605 
Dalmonech, L., Sant’Anna, J., Coimbra, P., \& Teixeira, A. (2008). Earnings Management e administração pública, 37 $A N P A D$, Rio de Janeiro. DOI: nd.

Dechow, P., Sloan, R., \& Sweeney, A. (1995). Detecting earnings management. Accounting Review, 70(2), 193-225. DOI: nd.

Ferreira, A., Carvalho, J., \& Pinho, F. (2013). Earnings management around zero: a motivation to local politican signalling competence. Public Management Review, 15, 657-686. DOI: https://doi.org/10.1080/14719037.20 12.707679

Flach, F. (2012). Falácia do déficit zero orçamentário. In: C. Mauss. Análise de demonstrações contábeis governamentais: instrumento de suporte à gestão pública. Atlas.

Gobetti, S. (2006). Ajuste fiscal nos estados: uma análise do periodo 1998-2006. Revista de Economia Contemporânea, 14(1), 113-140. DOI: nd.

Gobetti, S., \& Orair, R. (2017). Resultado primário e contabilidade criativa: reconstruindo as estatísticas fiscais "acima da linha" do governo geral. Texto para Discussão do IPEA (pp. 1-58), Rio de Janeiro, Brasil.

González, A., \& Velázquez, J. (2015). Estado explicativo del resultado presupuestario ajustado. Auditoría Pública, 65, 107-119. DOI: nd.

Healy, P. (1985). The effect of bonus schemes on accounting decisions. Journal of Accounting and Economics, 7, 85-107. DOI: https://doi.org/10.1016/0165-4101(85)90029-1

Hibbs, Jr. D. (1977). Political parties and macroeconomic policy. American Political Science Review, 71(4), 1467-1487. DOI: https://doi.org/10.1017/S0003055400269712

Jones, J. (1991). Earnings management during import relief investigations. Journal of Accounting Research, 29(2), 193-228. DOI: $10.2307 / 2491047$

Maltritz, D., \& Wüste, S. (2015). Determinants of budget deficits in Europe: The role and relations of fiscal rules, fiscal councils, creative accounting and the euro. Economic Modelling, 48, 222-236. DOI: https://doi.org/10.1016/j .econmod.2014.12.001

Milesi-Ferretti, G. (2003). Good, bad or ugly? On the effects of fiscal rules with creative accounting. Journal of Public Economics, 88, 377-394. DOI: https://doi.org/10.1016/S0047-2727(02)00076-2

Milesi-Ferretti, G., \& Moriyama, K. (2006). Fiscal adjustment in EU countries: A balance sheet approach. Journal of Banking \& Finance, 30, 3281-3298. DOI: https://doi.org/10.1016/j.jbankfin.2006.05.010

Nordhaus, W. (1975). The Political Business Cycle. Review of Economic Studies, 42(2), 169-190. DOI: $10.2307 / 2296528$

Paganotto, J., Oliveira, J., \& Antunes, G. (2017). Gerenciamento do resultado primário brasileiro: análise dos dividendos pagos por BNDES e CEF ao Tesouro Nacional. Revista Universo Contábil, 13(2), 65-88. DOI:10.4270/ruc.2017209

Pellicer, M., Hodges, R., \& Martí, C. (2013). Earnings management in English local governments: determining factors and instruments. In: XVII Congreso AECA, Pamplona, España.

Pennings, P., Keman, H., \& Kleinnijenhuis, J. (2006). Doing research in political Science, 2th. London: SAGE Publishing.

Pereira, P. (1997). A teoria da escolha pública (publi choice): uma abordagem neoliberal? Análise Social, 32(141), 419-442. Retrieved from http://www.jstor.org/stable/41011271

Persson, T. \& Tabellini, G. (1990). Macroeconomic policy, credibility and politics. Londres: Harvood Academic Publishers.

Queiroz, I., \& Rodrigues, A. (2014). Um estudo sobre o gerenciamento da informação contábil pelos municípios fluminenses para alcance do piso constitucional de aplicação em saúde. Pensar Contábil, 16(59), 4-9. DOI: nd.

Queiroz, I., Macedo, M., Rodrigues, A., \& Sauerbronn, F. (2013). Gerenciamento da informação contábil nos municípios brasileiros: a Lei de Responsabilidade Fiscal (LRF) e o limite de despesas consolidadas de pessoal. In: XIII Congresso USP de Controladoria e Contabilidade, São Paulo, Brazil. 
Reischmann, M. (2015). Creative accounting and electoral motives: Evidence from OECD countries. Ifo Working paper, No. 201, July.

Rogoff, K. \& Sibert, A. (1988). Elections and Macroeconomic Policy Cycles. Review of Economic Studies, 55(181), 1-16. DOI: $10.3386 /$ w 1838

Rogoff, K. (1990). Equilibrium political budget cycles. American Economic Review, 80(1), 21-36. DOI: 10.3386/ w2428

Rosenberg, J. (1992). Rationality and the Political Business Cycle: The case of local government. Public Choice, 73, 71-81. DOI: https://doi.org/10.1007/BF00142917

Santos, P., Machado, D., \& Scarpin, J. (2012). Gerenciamento de resultados no setor público: análise por meio das contas orçamentárias outras receitas e despesas correntes dos municípios de Santa Catarina. Revista de Contabilidade Vista \& Revista, 23(4), 15-43. DOI: nd.

Santos, S. (2007). O impacto da lei de Responsabilidade Fiscal nos orçamentos: uma análise do desempenho financeiro dos municípios do Rio Grande do Sul de 1997 a 2004. Master's thesis. Universidade do Vale do Rio dos Sinos, São Leopoldo, Brazil.

Santos, S., \& Alves, T. (2011). O impacto da lei de Responsabilidade Fiscal nos orçamentos: uma análise do desempenho financeiro dos municípios do Rio Grande do Sul de 1997 a 2004. Revista de Administração Pública, 45(1), 181-208. DOI: http://dx.doi.org/10.1590/S0034-76122011000100009.

Scaff, F., \& Athias, D. (2016). Dívida pública e desenvolvimento: do equilíbrio orçamentário à sustentabilidade financeira. Revista Cientifica Fagoc Jurídica, 1, 85-96. DOI: nd.

Schipper, K. (1989). Earnings management. Accounting Horizons, 3(4), 91-102. DOI: nd.

Souza, S. (2008). A fria austeridade das regras fiscais resiste ao calor das urnas? Oportunismo fiscal e contabilidade criativa nos estados brasileiros. PhD thesis. Universidade Federal de Pernambuco, Recife, Brasil.

Stalebrink, O. (2002). Governmental accounting \& financial reporting in transition: A study of "earnings management" under the accrual \& consolidation model. PhD thesis. George Mason University, Virgínia, EUA.

Stalebrink, O. (2007). An investigation of discretionary accruals and surplus-deficit management: Evidence from Swedish municipalities. Financial Accountability \& Management, 23(4), 267-424. DOI: https://doi.org/10.11 $11 / j .1468-0408.2007 .00437 . x$

Veiga, L. \& Veiga, F. (2005). Eleitoralismo nos municípios portugueses. Análise Social, 50(177), 865-889, DOI: nd, h ttps://www.jstor.org/stable/41012236.

Von-Hagen, J., \& Wolff, G. (2006). What do deficits tell us about debt? Empirical evidence on creative accounting with fiscal rules in the EU. Journal of Banking \& Finance, 30, 3259-3279. DOI: doi:10.1016/j.jbankfin.2006.05.011

\section{Notas}

1 La arena pública (metafóricamente) es una escena donde, ante los espectadores, se enfrentan los pretendientes a la "propiedad" (ownership) de problemas públicos. Esta metáfora de la "propiedad" incluye caracteres de control, de exclusividad y de transferabilidad (Cefaï, 2002, p. 8). Así, se puede decir que el presupuesto es una arena donde políticos disputan recursos públicos.

*Artículo de investigación científica y tecnológica.

\section{Licencia Creative Commons CC BY 4.0}

Para citar este artículo: Almeida-Santos, P., Pires da Silva, J., Matias-Pereira, J., \& Ferreira dos Santos, A. C. (2018). Evidencias de manipulación de resultados presupuestarios en el sector público: análisis crosssubnational para el Brasil. Cuadernos de Contabilidad, 19(48), 1-14. https://doi.org/10.11144/Javeriana. cc19-48.emrp 